\title{
Cystic isolated lymphoma of the base of the tongue: A case report
}

\author{
EDA SIMSEK ${ }^{1}$, HILAL KIZILTUNC OZMEN $^{2}$, YUSUF BILEN $^{3}$, MUHAMMED CALIK $^{4}$, \\ BETÜL GÜNDOGDU ${ }^{4}$ and BURAK ERDEMCI ${ }^{2}$ \\ ${ }^{1}$ Ear, Nose and Throat Clinic, Education and Research Hospital, Erzurum 25240; Departments of ${ }^{2}$ Radiation Oncology,
${ }^{3}$ Hematology and Oncology, and ${ }^{4}$ Pathology, Ataturk University Medical School, Erzurum 25170, Turkey
}

Received December 15, 2014; Accepted January 13, 2016

DOI: $10.3892 / \mathrm{ol} .2016 .4224$

\begin{abstract}
Isolated lymphoma of the base of the tongue is a relatively rare condition. The clinical presentation may vary from a submucosal mass with a cystic appearance to an ulcerated lesion. In the present case, a cystic mass was identified in a patient admitted with a sore throat. Pathological examination confirmed diffuse large B-cell lymphoma. The clinical and pathological picture of lymphomas covers a wide spectrum and the rate of occurrence in the oral cavity is 3-5\%. Among all malignant tumors of the oral cavity, lymphomas represent the third most common type of tumor, following squamous cell carcinoma and salivary gland neoplasms. Lymphomas of the oral cavity mostly arise from the Waldeyer ring, which is a lymphoid tissue. In our case, no recurrence was observed during the 8-month follow-up period after the patient was administered chemotherapy followed by radiotherapy. In conclusion, although isolated lymphoma of the base of the tongue is extremely rare, it should always be considered in patients presenting with a sore throat and the sensation of a foreign body in the throat.
\end{abstract}

\section{Introduction}

The clinical and pathological picture of lymphomas covers a wide spectrum, and the rate of occurrence in the oral cavity is $3-5 \%$ (1). Among the malignant tumors of the oral cavity, lymphomas represent the third most common type of tumor after squamous cell carcinoma and salivary gland neoplasms. Lymphomas of the oral cavity mostly arise from the Waldeyer ring, a lymphoid tissue, and rarely involve the hard palate, vestibulum and gingival tissue $(1,2)$. The most common symptoms of lymphoma include pain due to swelling, difficulty in speech due to ulceration and difficulty when swallowing. Physical examination may reveal a submucosal mass, a polypoid mass covered with a smooth mucosa or an ulcerated

Correspondence to: Dr Hilal Kiziltunc Ozmen, Department of Radiation Oncology, Ataturk University Medical School, Erzurum 25170, Turkey

E-mail: hkiziltuncozmen@hotmail.com

Key words: isolated lymphoma, radiotherapy, chemotherapy lesion (3). The diagnosis may be confirmed by pathological examination. The treatment of lymphomas depends on the stage of tumor, the histological type and the age of the patient, and may involve chemotherapy, radiotherapy or combined therapy $(4,5)$. In this study, the case of cystic isolated lymphoma of the base of the tongue in a 48 -year-old male who presented with a sore throat is reported. Written informed consent was obtained from the patient.

\section{Case report}

Patient presentation. In May 2013, a 48-year-old male patient was admitted to the Ear, Nose and Throat clinic of the Education and Research Hospital (Erzurum, Turkey) with the complaint of pain for 2 months and the sensation of a foreign body in the throat. Medical history revealed that the patient had received antibiotic treatment several times for these complaints but no improvement had been noted. His family history revealed no genetic disorders. The patient was a non-smoker and no alcohol use was noted.

Examinations and findings. An endoscopic laryngeal examination revealed a $1.5 \times 2 \mathrm{~cm}$ cystic mass with a wide base and smooth mucosal cover on the right side of the tongue base extending to the vallecular. Other laryngeal structures were normal. No cervical lymphadenopathy was identified by palpation. Findings from other systemic and head and neck examinations were within the normal limits. Neck magnetic resonance imaging was performed using a MAGNETOM Avanto 1.5T device (Siemens Aktiengesellschaft, Munich Germany), which revealed a 20x17x16 mm lesion with a cystic component and peripheral minimal contrast uptake (Fig. 1). The complete blood count results were as follows: hemoglobin, $15 \mathrm{~g} / \mathrm{dl}$; hematocrit, $45.5 \%$; white blood cells, $7.4 \times 10^{3} / \mathrm{ml}$; red cell distribution width, 13.7 ; platelets, $316 \times 10^{3} / \mathrm{ml}$. ELISA tests for hepatitis and HIV were negative. The chest X-ray was normal.

Surgery and diagnosis. Under general anesthesia, the mass with its cystic component extending to the vallecular was excised and wide incisional biopsy materials were obtained from the vegetative field of the cyst base. Pathological and immunophenotypic examination revealed that CD20, CD19, Bcl-2 and CD10 were positive whereas CD3 was negative. The Ki-67 proliferation index was $85 \%$. 

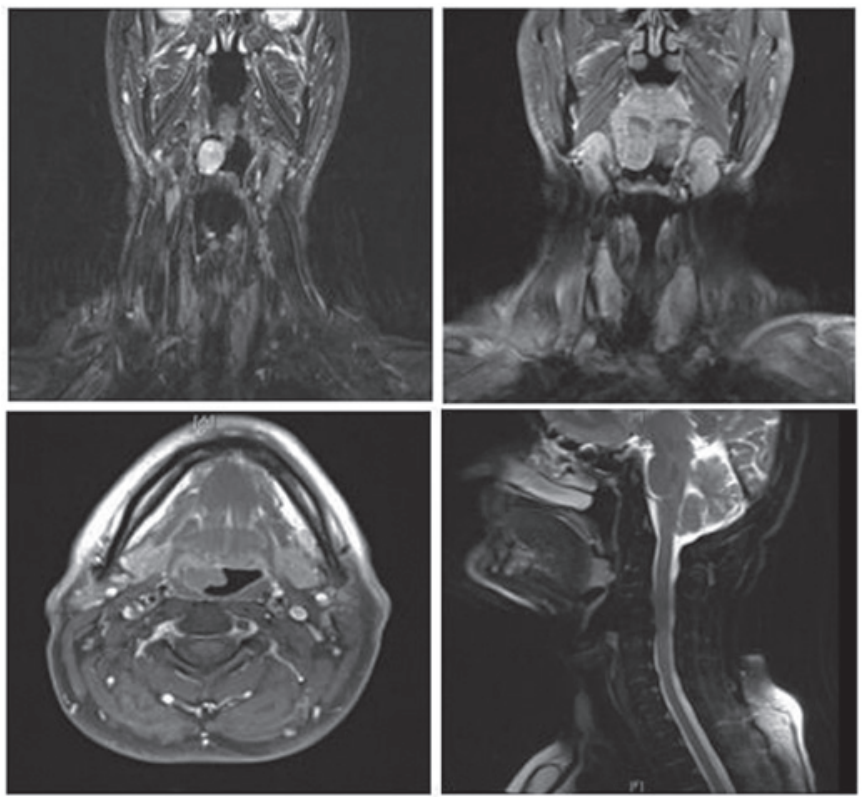

Figure 1. Neck magnetic resonance imaging of the preoperative tumor. Top left and right, coronal images; bottom left, transverse image; bottom right, sagittal image.

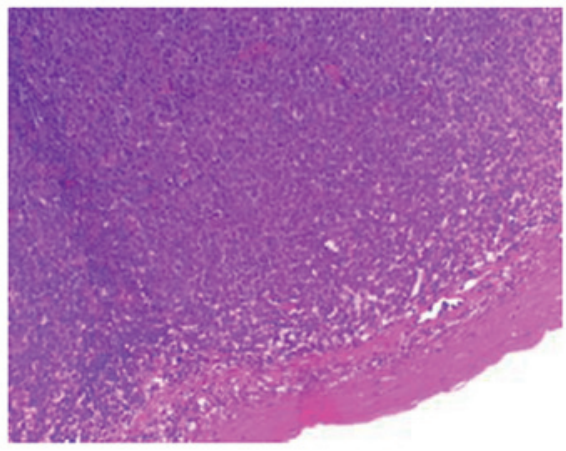

Figure 2. Diffuse infiltration of round and uniform tumor cells was observed in the tonsillectomy specimen (hematoxylin and eosin staining; magnification, $\mathrm{x} 200)$.

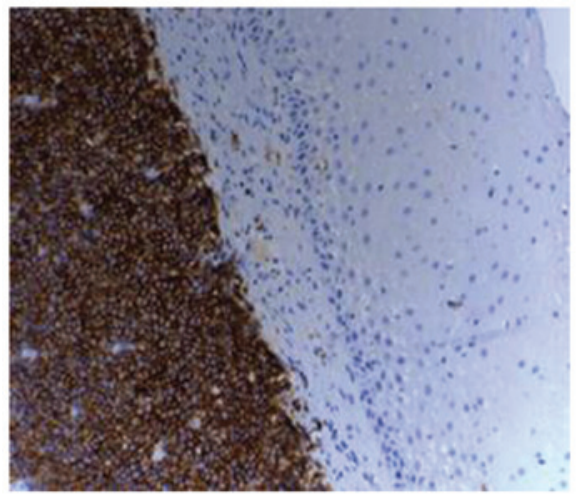

Figure 3. Immunohistochemically, tumor cells were strongly positive for CD20 (magnification, x200)

Pathological examination confirmed high-grade diffuse large B-cell lymphoma (DLBCL; Figs. 2-4). Pre-treatment positron emission tomography-computed tomography (PET-CT) using

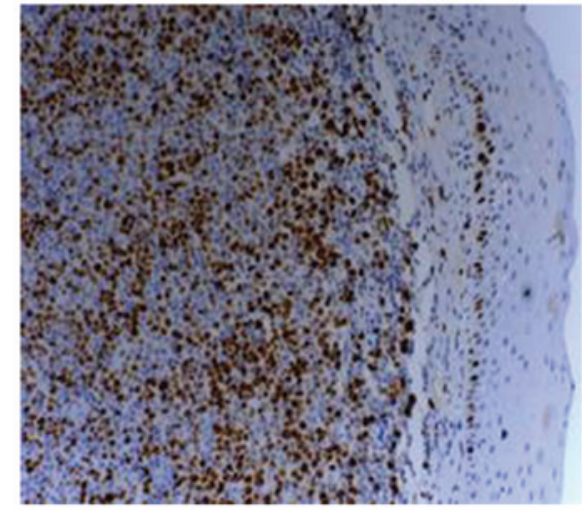

Figure 4. Immunohistochemically, tumor cells had a high positive Ki-67 index (magnification, $\mathrm{x} 200$ ).
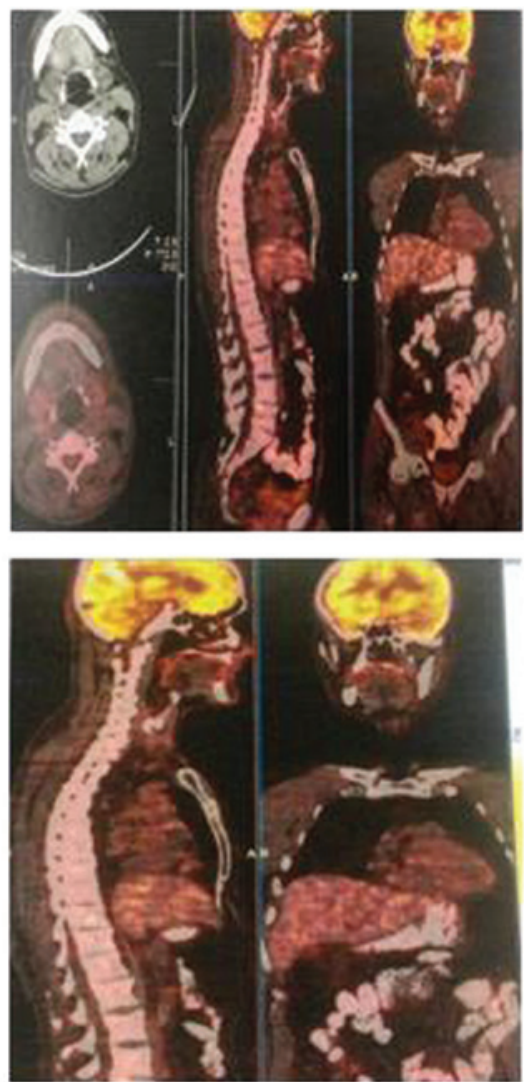

Figure 5. Preoperative positron emission tomography-computed tomography images of the tumor. Top left, axial image; top middle, sagittal image; top right, coronal image; bottom left, sagittal image; and bottom right, coronal image.

a Siemens Biograph system (Siemens, Knoxville, TN, USA) demonstrated no focal involvement (Fig. 5). The patient was diagnosed with grade 1B DLBCL.

Treatment and follow-up. A four-session rituximab (675 mg/day on day 1$)$, cyclophosphamide $(1,350 \mathrm{mg} /$ day on day 1$)$, doxorubicin $(90 \mathrm{mg} /$ day on day 1$)$, oncovin $(1.4 \mathrm{mg} /$ day on day 1$)$ and prednisolone (90 mg/day on days 1-5) (R-CHOP) multiple chemotherapy regimen followed by involved-field regional radiotherapy (IFRT) were planned. During the following 
3 months, the four-session (once over a 21 day period repeated 4 times) R-CHOP chemotherapy and IFRT treatments were administered without any complications. Follow-up PET-CT imaging revealed no involvement. No recurrence of the disease was observed during the 8-month follow-up period.

\section{Discussion}

As a lymphoproliferative disease, the occurrence rate of lymphomas in the oral cavity is $3-5 \%$ among all oral malignancies (1). The most common location of lymphomas in the oral cavity is Waldeyer's lymphatic ring, followed by the hard palate, vestibulum and alveoli $(3,5)$. In Waldeyer's ring, tonsillary $(50 \%)$, nasopharyngeal and tongue base involvement is the most common (5). Isolated non-Hodgkin lymphoma of the tongue base is extremely rare and the clinical appearance may be a submucosal mass, a polypoid mass covered with a smooth mucosa or a ulcerated lesion $(3,6)$. In our case, the tumor was isolated to the tongue base and covered by a smooth mucosa.

Symptoms of oral lymphomas include pain due to the effect of the mass, difficulty in speech, and dysphagy or odinophagy due to ulceration $(3,5)$. The so-called B-symptoms, including fever, night sweats and weight loss, are fewer in non-Hodgkin lymphoma compared with Hodgkin lymphoma, and may not be observed at all (5). In our case, there were no symptoms apart from sore a throat and the sensation of a foreign body.

There are distinct approaches in the treatment of oral cavity non-Hodgkin lymphomas. Certain studies suggest multiple chemotherapy combined with radiotherapy for primary lymphomas of Waldeyer's ring $(2,5,7)$. A combined chemotherapy and radiotherapy treatment was also administered in the present case, and 8 months of follow-up revealed no recurrence.

In conclusion, although isolated lymphoma of the base of the tongue is extremely rare, it should always be considered in patients presenting with a sore throat and the sensation of a foreign body in the throat. A tongue base examination should also be performed. Polypoid and cystic masses covered with a smooth mucosa may mimic benign lesions; thus, lymphoma should be considered in the differential diagnosis.

\section{References}

1. Lim HJ, Lim JY, Kim YM, Kim CS, Choi SJ, Yi HG, Choi WG and Lee MH: Primary diffuse large B cell lymphoma of the base of tongue. J Cancer Res Ther 8: 135-137, 2012.

2. Guastafierro S, Falcone U, Celentano M, Cappabianca S, Giudice A and Colella G: Primary mantle-cell non-Hodgkin's lymphoma of the tongue. Int J Hematol 88: 206-208, 2008.

3. Hmidi M, Touiheme N, Elboukhari A, Kettani M, Elmejareb C and Messary A: Primary B cell lymphoma of the tongue: a case report. Pan Afr Med J 12: 5, 2012.

4. Terada T: Primary non-Hodgkin B-cell lymphoma of the tongue. Br J Oral Maxillofac Surg 49: e18-e19, 2011.

5. Tarhan AE, Akdoğan MV, Haberal AN, Özlüoğlu LN and Demirhan B: Lymphoma presenting as hypopharynx and tongue base malignancy. KBB-Forum 3: 54-58, 2004 (In Turkish).

6. Jovanovic MB: An exophytic localized lymphoma of the tongue base. Otolaryngol Head Neck Surg 139: 468-469, 2008.

7. Ezzat AA, Ibrahim EM, El Weshi AN, Khafaga YM, AlJurf M, Martin JM, Ajarim DS, Bazarbashi SN, Stuart RK and Zucca E: Localized non-Hodgkin's lymphoma of Waldeyer's ring: clinical features, management and prognosis of 130 adult patients. Head Neck 23: 547-558, 2001. 